\title{
USO DE LEGUMINOSAS FLORESTAIS NODULADAS E MICORRIZADAS COMO AGENTES DE RECUPERAÇÃO E MANUTENÇÃO DA VIDA DO SOLO: UM MODELO TECNOLÓGICO
}

FRANCO, A.A., DIAS, L.E, FARIA, S.M. DE, CAMPELLO, E.F.C. \& SILTA. E.M.R. DA

\section{Resumo:}

Diversas espécies de leguminosas florestais apresentam a capacidade de se associarem sinbioticamente com bactérias que fixam nitrogênio atmosfërico e ao mesmo tempo a fungos micorrizicos que aumentam a eficiência do uso da água e nutrientes do solo, especialmente o fósforo. Trabalhos de levantamento e seleção de estirpes de rizóbio e de fungos micorrizicos, foram conduzidos para a obtenção de microrganismos eficientes para espécies leguminosas que apresentam rápido crescimento, mesmo sob condições de estresse ambiental. Este trabalho apresenta um modelo de recuperação de solos degradados usando estas espécies leguminosas associadas a microsimbiontes juntamente com fomecimento de $\mathrm{P}, \mathrm{K}, \mathrm{S}, \mathrm{Ca}, \mathrm{Mg}$ a micronutrientes na forma de adubos baratos e de disponibilidade a longo prazo. O modelo proposto baseia-sa no fato que o aporte de grandes quantidades de matéria orgânica com baixa relação $\mathrm{C} / \mathrm{N}$, além da proteção do solo contra o impacto da chuva e controle da erosão, acelera a ciclagem de nutrientes e o retorno da vida do solo. Diversos ensaios em andamento têm mostrado a viabilidade desta técnica, onde na região Sudeste têm se destacado as espécies: Acacia mangium. A. anriculiformis. A. holocericea, Albizia saman, $A$. guachapelle, Enterolobium contortisiliqutu, Mimosa caesaipinifolia, M. scabrella, M. tenuiflora, Clitoria fairchildiana, Leucaena lencocephala e Paraserianthes falcataria e na região Amazônica, além das Acacias, $C$. fairchildiana e E. contortisiliqutum, têm-se destacado o Stryphnodendrum adstringens e Sclerolobium paniculatum.

\section{Abstract: \\ "Use of nodulate and mycorrizal forest leguminous trees as agents of recovery an maintenance of soil life: a technological model"}

Several leguminous trees are able to tix atmospheric nitrogen when in symbiosis with rhizobia and to form nycorrhizal associations which improve the efficiency of the absorption of water and mineral nutrients from the soil, especially phosphorus, the most important and limiting nutrient in tropical soils. Several experiments conducted at the National Research Center of Agrobiology (CNPAB), have identified efficient rhizobial and endomycorrhizal symbionts for several fast growing legume trees, which are adapted to stress conditions. These species when associated with the microsymbionts have been shown to be suitable for revegetation of degraded tropical acid soils. when the deficiencies of the major nutrients, other than nitrogen. have been corrected. The addition of a large amount of organic matter (litter), with low C N ratio, besides protecting the soil from the direct impact of the raindrops and erosion, accelerates the eycling of soil nutrients and favours the return of life to the soil. In the Southeast region of Brazil the most promising species were found to be: Acacia mangitum. A. anriculiformils, A. holocericea, Albizla saman. A. 
guachapelle, Enterolobium contorisiliquum, Mimosa caesalpinifolia, M. scabrella, $M$. tenufflora, Chitoria fairchildiana, Leucaena leucocephala and Paraserianthes faicataria. In the Amazonian region, besides the Acacias, C. fairchildiana and E. contortisiligum, other species such as Stryphnodendrum adstringens and Sclerolobium paniculatum have shown good potential for revegetation of degraded soils.

\section{Introdução}

O inicio da exploração agricola das regiões próximas da costa brasileira e fronteiras agricolas surgidas ao longo deste século, baseou-se na agricultura itinerante ou migratória. Este modelo agricola, é muito dependente da fertilidade natural do solo. Como a maioria dos solos brasileiros apresenta baixa fertilidade natural, o rápido esgotamento de nutrientes essenciais, condicionou o caráter migratório da exploração agricola e como consequêencia resultou no aparecimento de rerras degradadas. Em todo o mundo, estima-se que a cada ano cerca de 1,1 milhões de ha de florestas tropicais. secundárias e energéticas, sejam destruidas ou seriamente degradadas peia expansão agricola (WRU/IRF, 1988 , citado por PARROTA, 1992). Em épocas mais recentes em várias regiões do Brasil, este modelo ainda tem sido utilizado.

Além das áreas de exploração agrícola. ações antrópicas mais drásticas ao solo têm sido observadas como nas áreas de mineração, construção de barragens, estradas, ferrovias, etc. Em áreas de mineração, além da retirada da vegetação natural observa-se uma intensa movimentação do solo na abertura da lavra e em alguns casos, acrescidas de um considerável volume de rejeitos que também contribuem para o distúrbio da área.

Em todas essas situações, o ponto em comum e o mais critico é a remoção dos horizontes superficiais do solo. A remoção da matéria orgânica contida nessa camada, além de causar sérios problemas na estrutura, disponibilidade de água e atividade biológica do solo, prejudica o suprimento de nutrientes essenciais como $\mathrm{P}, \mathrm{S}$ e principalmente de $\mathrm{N}$ às plantas.

Neste sentido, a estratégia de recuperação de solos degradados deve basear-se numa tecnologia que promova não apenas a utilização de espécies de rảpido crescimento, mas também que sejam capazes de melhorar o solo através do aporte de matéria orgânica. Esta melhoria se faz tanto pela deposição de material vegetal como pela reciclagem de nutrientes (MONTAGNINI \& SANCHO, 1990). Dessa forma, além de formar cobertura vegetal protetora do solo, também, facilita - posterior estabelecimento de outras espécies vegetais mais exigentes (BAGGIO, 1986).

A EMBRAPA/CNPAB vem realizando estudos com 0 objetivo de definir soluções técnico-cientificas, baseadas na utilização de espécies leguminosas, para a contenção de áreas de riscos e a 
recuperação de solos degradados (FRANCO et al., 1991). O presente trabalho tem como objetivo apresentar e discutir o modelo tecnológico que vem sendo utilizado e os resultados já alcançados.

\section{Fundamentos e Princípios do Modelo Tecnológico}

A revegetação de áreas degradadas geralmente é feita com alto investimento, muitas vezes às custas da transferência da camada fértil de outras áreas, ou com grande aporte de matéria orgânica e/ou fertilizantes. A tecnologia proposta pela EMBRAPA/CNPAB viabiliza à baixo custo, a recuperação de solos, através da utilização de espécies leguminosas noduladas e micorrizadas. Quando necessário, corrige-se a deficiência dos demais nutrientes com produtos de baixo custo, como gesso, fosfato de rocha e quando disponível, composto orgânico (FRANCO et al., 1991).

Dentre as diferentes espécies florestais que podem ser utilizadas na recuperação de solos, as leguminosas capazes de formar simbiose eficiente com rizóbio despertam grande interesse (ALLEN \& ALLEN, 1981; FARIA et al., 1989). Essas espécies, quando associadas a fungos micorrízicos, ainda propiciam melhor aproveitamento de fósforo e outros nutrientes como $\mathrm{Zn}, \mathrm{Mn}$ e Cu (LAMBERT et al., 1979; MANJUNATH \& HABTE, 1988; SIQUEIRA \& FRANCO, 1988). Grande parte dessas espécies apresenta uma elevada produção de biomassa com significativo aporte de folhas ao solo. Estimativas na região nordeste, indicam que 5,8 t/ha.ano de material de sabiá (Mimosa caesalpiniifolia). podem retornar ao solo (SUASSUNA, 1982). Em condições climáticas similares. a Leucaena leucocephala contribui com um aporte na ordem de 10 tha. ano (SANDHU et al., 1990). Em sistema de sombreamento para café na América Central, a Ervthrina sp. contribue com um aporte de até 13,5 t/ha.ano de material vegetal (GLOVER \& BEER, 1986). Desta forma as espécies fixadoras de nitrogênio podem apresentar uma significativa melhoria na fertilidade do solo.

Além do volume de material vegetal que é adicionado ao solo, as características desse material são de grande importância para a fertilidade do solo. As leguminosas são geralmente ricas em nitrogênio apresentando baixa relação $\mathrm{C} / \mathrm{N}$. Desta forma a liberação de nutrientes de resíduos é geralmente mais rápida que a de plantas com baixo teor de nitrogênio (alta relação $\mathrm{C} / \mathrm{N}$ ). Entretanto, há diferenças mesmo entre as plantas com baixa relação $\mathrm{C} / \mathrm{N}$, sendo mais rápida em resíduos de Erythrina que de Inga edulis ou Cajanus cajan (PALM, 1988, citado por SZOTT et al., 1991), em leucena ou sabiá do que de A. mangium. Da mesma forma, a velocidade de liberação de fósforo, potássio, enxofre, cálcio, magnésio e micronutrientes também varia com as espécies. O "litter" produzido por Leucaena leucocephala pode apresentar "turn-over" de nitrogênio inferior a um ano, e com uma liberação anual de nitrogênio da ordem de $200 \mathrm{~kg} / \mathrm{ha}$ (SANDHU et al., 1990). 
Em terrenos de declividade acentuada, além da incorporação de material orgánico, deve-se lançar mão de espécies com muitas raízes laterais e que promovam rápida cobertura do solo. protegendo-o dos agentes de erosão Desta Corma, a escolha da espécie de leguminosa a ser utilizada pode ser otimizada conforme as características do terreno. clima e objetivos imediatos da revegetação.

A EMBRAPA/CNPAB rem realizando pesquisas a fim de identificar espécies nativas ou introduzidas com potencial para revegetação de solos degradados. Vem desenvolvendo tambem estudos para selecionar as estirpes de rizóbio mais eficientes na fixação de nitrogênio para essas espécie (FARIA et ai.. 1984a, 1987, 1991). Em levantamentos realizados nas regiões Sudeste, Norte e Nordeste 635 espécies foram diagnosticadas com capacidade de fixar nitrogênio. sendo que muitas destas demonstram potencial produtivo para diversos fins (FARIA et al., 1984b, 1987, 1989).

DIAS (1985), ao descrever o trabalho de reabilitação de áreas mineradas pela extração de bauxita em Poços de Caldas-MG, cita a elevada acidez. e concentração de alumínio trocável do solo como um dos principais problemas a ser enfrentado. A acidez é na realidade generalizada na maioria dos solos tropicais (MUNNS \& FRANCO, 1982). Neste sentido, e lerando em consideração que esta limilação é agravada em grande parte dos solos degradados, a EMBRAPA/CNPAB tem realizado pesquisas que visam selecionar estirpes de rizóbio capazes de crescerem em condições de baixo $\mathrm{pH} c$ de toxidez de alumínio (SILVA \& FRANCO, 1984; OLIVEIRA \& FRANCO, 1988).

Outra característica comum dos solos degradados, como conseqüência da ausência de vegetação é o aumento da temperatura do solo. Para PEREIRA \& KNOWLES (1985), este é um dos principais problemas enfrentados na execução do programa de reabilitação das áreas mineradas em Porto Trómbetas, PA.

De mancira geral, o funcionamento da simbiose rizóbio/planta é mais sensivel ao efeito de altas temperaturas, do que quando as plantas são supridas com nitrogênio mineral. Por outro lado. solos tropicais, as temperaturas nos $5 \mathrm{~cm}$ superficiais, freqüentemente atingem valores superiores a $40^{\circ} \mathrm{C}$ (Eaglesham \& Ayanaba, 1984; Mercante, 1993). Assim, tom-se dado ênfase também na busca de espécies de plantas e estirpes de rizóbio mais adaptadas a estas condições (CUNHA \& FRANCO, 1988).

A grande maioria das espécies florestais, quando na sua fase de muda, necessita de maior disponibilidade de fósforo pois a demanda deste nutriente é mais intensa (NOVAIS et al, 1990). As leguminosas dependendo da simbiose como fonte de nitrogênio, requerem alto teor de fósforo no solo para suprir as necessidades adicionais dos nódulos. Além disto a presença de nódulos pode diminuir o crescimento de raizes quando comparadas com plantas que recebem o $\mathrm{N}$ mineral (CASSMANN et al., 1981). 
Vários trabalhos têm mostrado resposta positiva das leguminosas à inoculação com rizóbio juntamente com micorrizas. O sabiá e a bracatinga (Mimosa scabrella) apresentam uma resposta significativa à inoculação com Gigaspora margarita (MONTEIRO, 1990).

A metodologia proposta pela EMBRAPA/CNPAB inclui a inoculação com fungos micorrizicos vesicular-arbusculares como uma maneira econômica de tornar as plantas mais eficientes na absorção de água e nutrientes do solo tais como fósforo, zinco, magnésio e cobre.

Em área pertencente a EMBRAPACNPAB em Itaguaí-RJ, foram testadas as espécies sabiá, Acacia auriculiformis, Cliricidia sepium e Eucalyptus grandis (testemunha não fixadora de nitrogênio) em solo podzólico vermelhoamarclo $(\mathrm{pH}=4,7)$, onde as camadas superiores haviam sido removidas para a construção de uma barragem. As mudas foram produzidas em substrato contendo $20 \%$ de fosfato de rocha e inoculadas com rizóbio específico e fungos micorrízicos vesicular-arbusculares. Em cada cova foi adicionado 1 litro de esterco de galinha curtido e $80 \mathrm{~g}$ de fosfato de rocha. Nesta área, foi utilizado espaçamento de $2 \mathrm{~m} \mathrm{x}$ $\mathrm{lm}$. O sabiá e a A. auriculiformis cobriram quase totalmente o solo já no $1^{\circ}$ ano enquanto a $G$. sepium apresentou crescimento menos rigoroso. O eucalipto apresentou crescimento satisfatório apenas no primeiro ano. enquanto durou o efeito da matéria orgânica adicionada.

No município de Piraí-RJ, foi conduzido um ensaio onde além do sabiá, foram testadas Acacia mangium, bracatinga, angico (Anadenanthera peregrina) e Eucaliptus grandis. O experimento foi conduzido em área de uni latossolo $(\mathrm{pH}=4,6)$, onde os horizontes superficiais haviam sido removidos. Após aproximadamente 6 meses no campo, as plantas dos tratamentos que não receberam matéria orgânica na cova apresentavam sintomas de deficiência de enxofre, que desapareceram com a aplicação de gesso agrícola. O sabiá e a A. mangium apresentaram bom desenvolvimento mesmo sem adição de matéria orgânica (Fig. 1). As copas cobriram $80 \%$ da área já no primeiro ano (espaçamento de $1 \mathrm{~m} \times 1 \mathrm{~m}$ ). Ao final do segundo ano observoul-se um completo recobrimento do solo atrarés da deposição de material vegetal, que passou a exercer proteção contra a erosão. Novamente, observou-se um crescimento satisfatório para o eucalipto apenas para as plantas que receberam a matéria orgânica na cova e enquanto perdurou o seu efeito.

Em Porto Trombetas-PA foi instalado um ensaio em área de mineração para extração de bauxita, onde os horizontes superficiais haviam sido removidos para permitir a extração do minério. Após a lavra. houve a reposição mecânica do subsolo desprovido de matéria orgânica, constituindo assim. um substrato com caracterísrticas físicas e químicas impróprias para crescimento vegetal. Para este experimento estão scndo araliadas as seguintes espécies: taxibranco (Sclerolobium paniculatum), Acacia mangium, Acacia auriculiformis, Enterolobium contortisiliqum, mari-mari (Cassia leiandra), Senna siamen, 
morototó (Didimopanax morototoni), camuze (Stryphnodendron adstringens), murici (Byasonina crassifolia) e cupiúba (Goupia glabra). O plantio foi realizado no final do periodo chuvoso, fato que permitiu que as plantas estivessem mais susceptiveis aos défices hídricos do período de estiagem. As espécies que apresentaram melhor desenvolvimento após 8 meses do plantio foram as leguminosas, notadamente as acácias e o camuze. A falta de estrutura do solo, aliada à deficiência hídrica na região, que apresenta alta taxa de evapotranspiração potencial, é que estão dificultando o estabelecimento das plantas, tendo sido o camuze a espécie menos afetada.

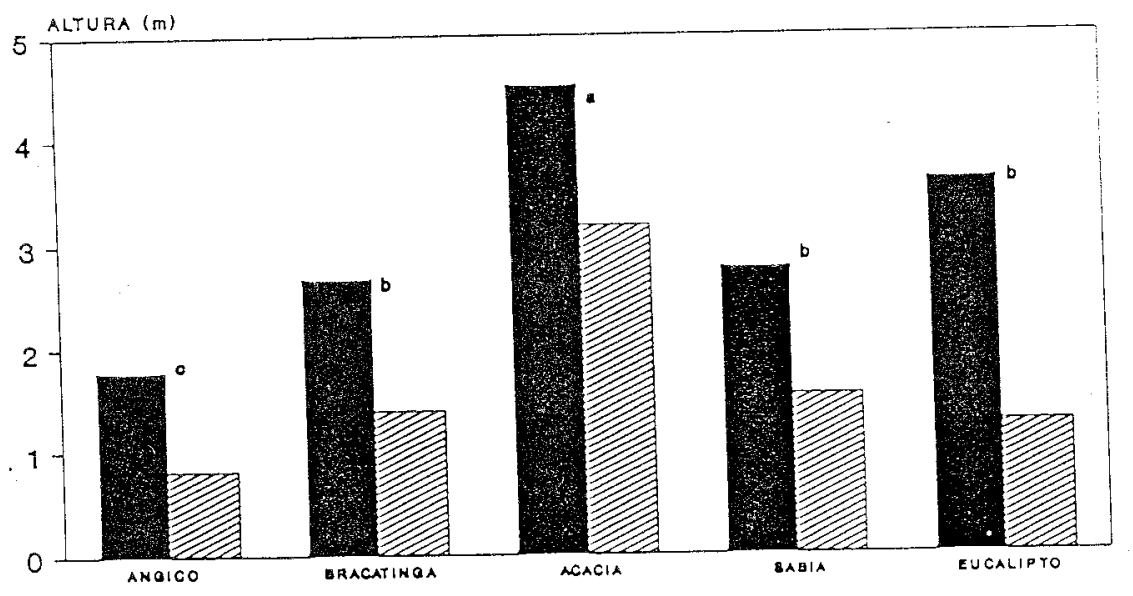

- Esterco - Esterco

FONTE: Franco et al, 1991.

Figura 1 - Altura aos 24 meses de leguminosas arbóreas e eucalipto, com e sem aplicação de esterco em latossolo decapitado.

As espécies com letras diferentes, diferem entre si pelo teste de tukey $p=0,05$, ccorrendo também diferenças significativas $(p=0,01)$ entre os tratamentos com e sem esterco).

\section{Conclusão}

A utilização de espécies leguminosas arbóreas fixadoras de nitrogênio atmosférico e micorrizada, aliada a adição de fosfato de rocha tem se mostrado cono uma técnica de grande viabilidade econômica e biológica para a recuperação de solos degradados. Para tanto são utilizadas mudas inoculadas com 
fungos micorrízicos vesicular-arbusculares e estirpes selecionadas de rizóbio, que asseguram uma maior capacidade de desenvolvimento destas espécies em solos sem os horizontes superficiais e com características físico-químicas desfavoráveis.

Diferentes ensaios em andamento permitem destacar para a região sudeste, entre outras espécies: Acacia mangium, $A$. auriculiformis, $A$. holocericea, Albizia saman, A. guachapelle, Enterolobium contortisiliquum, Mimosa caesalpiniifolia, M. scabrella, M. tenuiflora, Clitoria fairchildiana, Leucaena leucocephala e Paraserianthes falcataria. Para a região Norte, além das Acacias, C. fairchildiana e E. contortisiliquum, têm-se destacado 0 Stryphnodendrum adstrigens e Sclerolobium paniculatum.

\section{Bibliografia}

ALLEN, O.N., ALLEN, E.K. 1981. The Leguminosae. Madison, University of Wiscosin. 812p.

BAGGIO, A. 1986. O papel da silvicultura alternativa na proteção florestal. In: Congresso Florestal Brasileiro, 5. Olinda. Silvicultura, 41:55-57.

CASSMANN, K.G., WHITNEY, A.S., FOX, R.L. 1981. Phosphorus requirements of cowpea and soybeans as affected by mode of $\mathrm{N}$ nutrition. Agron. $\mathrm{J}_{\text {.. }}$ 73:17-22.

CUNHA, C.O., FRANCO, A.A. 1988. Efeito de altas temperaturas na nodulação e crescimento de 10 leguminosas arbóreas. An. Acad. Bras. Cienc. Rio de Janeiro, RJ.

DIAS, A.C. 1985. Reabilitação de áreas mineradas de bauxita. In: BRASIL, Ministério das Minas e Energia. Coletânea de trabalhos sobre controle ambiental na mineração. Brasília, Dep. Nacional de Produção Mineral, p. $175-183$.

EGLESHAM A.R.J., AYANABA, A. 1984. In: SUBBA RAO, W.S. (ed). Current development in biological nitrogen fixation. Oxford \& IBH Publishing Co. New Delhi, p.1.

FARIA, S.M.de, FRANCO, A.A., JESUS, R.M., MENANDRO, M.S., BAITELLO, J.B., MUCCI, E.S.F., DÖBEREINER, J., SPRENT, J.I. $1984 \mathrm{~b}$. New nodulation legume trees from South East Brazil. New Phytol. 98(2):317-328.

MOREIRA, V.G., FRANCO, A.A. 1984a. Seleção de estirpes de Rhizobium para leguminosas florestais. Pesq. Agrop. Bras.. 19(s/n):175-180. 
LIMA, H.C., FRANCO, A.A., MUCCI, E.S.F., SPRENT, J.I. 1987. Nodulation of legume trees from South East Brazil. Plant and Soil, 99:347-356.

CARVALHO, C.W.P., RAMOS, A.L.M.. FREIRE, M.F.I. GUIMARÃES. A.F. 1991. Obtenção e seleção de estirpes de Rhizobium spp para leguminosas arbóreas. Congresso Brasileiro de Ciência do Solo, 23. Porto Alegre, Julho, p. 196.

- LEWIS, G.P., SPRENT, J.I., SUTHERLAND, J.M. 1989. Occurrence of nodulation in the Leguminosae. New Phytol., 111:607-619.

FRANCO, A.A., CAMPOS NETO, D., CUNHA, C.O., CAMPELlO, E.F.C.. MONTEIRO, E.M. da S., SANTOS, C.J.F., FONTES, A.M., FARIA. S.M. de. 1991. Revegetação de solos degradados. $I n$ : Workshop sobre recuperação de áreas degradadas, 1. 1990. Ptaguaí. Anais... UFRRJ/Departamento de Ciências Ambientais. p.133-157.

GLOVER, N., BEER, J. 1986. Nutrient cycling in two traditional central american agroforestry. Agrof. Syst., 4:77-87.

LAMBERT, D.H., BAKER, D.E., COLE Jr., H. 1979. The role of mycorrhiza in the interactions of phosphorus with zinc, cooper and other elements. Soil Sci. Soc. Amer. I. 43:976-980

MANJUNATH, A., HABTE, M. 1988. Development of vesicular-arbuscular mycorrhizal infection and uptake of immobile nutrients in Leucaena leucocephala. Plant and Soil, 106:97-103.

MERCANTE, F. 1993. Uso de Leucaena leucocephaia na obtenção de Rhizobium tolerante à temperatura elevada para inoculação do feijociro. Dissertação de Mestrado. UFRRJ. 149p.

MONTAGNINI, F., SANCHO, F. 1990. Impacts of native trees on tropical soils: a study in the Atlantic lowlands of Costa Rica, Central America. Ambio. 19:386-390.

MONTEIRO, E.M. da S. 1990. Resposta de leguminosas arbóreas à inoculação com rizóbio e fungos micorrizicos vesículo-arbusculares em solo ácido. Itaguaí. Tese de doutorado. UFRRJ. $221 \mathrm{p}$.

MUNNS, D.N., FRANCO, A.A. 1982. Soil constraints to legume production. In: GRAHAM , P., HARRIS, S.C. (eds). Biological Nitrogen Fixation Technology for Tropical Agriculture. Papers presented at a workshop held at CIAT. March 9-13, 1981. Cali, Colombia. CIAT p. 133-152.

NOVAIS, R.F.. BARROS, N.F., NEVES, J.C.L. 1990. Nutrição Mineral do Eucalipto. In: BARROS, N.F., NOVAIS. R.F. (eds). Relação soloeucalipto. Viçosa. Folha de Viçosa. p.25-98. 
OLIVEIRA, E., FRANCO, A.A. 1988. Efeito do pH do solo na nodulação, fixação de $\mathrm{N}$ e crescimento de 4 espécies leguminosas arbóreas. An. Acad. Bras. Cien. Rio de Janeiro.

PARROTA, J.A. 1992. The rolc of plantation forest in rehabilitating degraded tropical ecosystems. Agriculture. Ecosystems and Environment. 41:115-133.

PEREIRA. F.S., KNOWLES, O.H. 1985. Recuperação das áreas mineradas pela Mineração do Rio do Norte em. Porto Trombetas-PA. In: BRASIL, Ministério das Minas e Energia. Coletânea de trabalhos sobre controle ambiental na mineração. Brasilia. Dep. Nacional de Produção Mineral, p. $343-358$.

SANDHU, J., SINHA, M., AMBASHT., R.S. 1990. Nitrogen release from decomposing litter of Leucaena leucocephala in the dry tropics. Soil Biol.. Biochem. 22(6):859-863.

SILVA, G.G., FRANCO, A.A. 1984. Seleção de estirpes de Rhizobium spp de leguminosas florestais em meio de cultura tolerantes à acidez e à toxidez de Al. Pesq. Agrop. Brasil, 19(s/n):169-173.

SIQUEIRA, J.O.. FRANCO, A.A. 1988. Biotecnologia de solo: Fundamentos $\underline{\mathrm{e}}$ perspectivas. Brasília: MEC/ABEAS: Lavras, ESAL/FAEPE, 235p.

SUASSUNA, J. 1982. Efeitos da associação do sabiá (Mimosa caesalpinaefolia. Benth) no comportamento do jacarandá (Dalbergia nigra FR. Allen) e da peroba branca (Tabebuia stenocalyx, Sprague \& Stapf) na zona da mata de Pernambuco, Recife. Dissertação de Mestrado. UFRPe, 179p.

SZOTT. L.T., FERNANDES, E.C.M., SANCHES. P.A. 1991. Soil plant interactions in agroforestry systems. Forest Ecology and Management. $\pm 5: 127-152$.

\section{Endereços:}

FRANCO, A.A. FARIA, S.M. DE, CAMPELLO, E.F.C. \& SILVA, E.M.R. DA EMBRAPA - Centro Nacional de Pesquisa de Agrobiologia

Fax: 021 682-1230 - Cx. Postal 74505 - CEP. 23851-970 - Seropídica - Itaguaí - R.

DIAS, L.E.

Universidade Federal de Viçosa - Departamento de Solos

Av. Peter Henry Rolfs, s n ${ }^{\circ}$ - Campus Universitario - FAX (031) 899-2648

CEP 36570-000 - VIÇOSA - MG 